THE ASTROPHYSICAL JouRNAL, 369:L35-L40, 1991 March 10

(C) 1991. The American Astronomical Society. All rights reserved. Printed in U.S.A.

\title{
STELLAR PHOTOMETRY WITH THE HUBBLE SPACE TELESCOPE WIDE-FIELD/PLANETARY CAMERA: A PROGRESS REPORT
}

\author{
Jon A. Holtzman, ${ }^{1}$ Edward J. Groth, ${ }^{2}$ Robert M. Light, ${ }^{3}$ S. M. Faber, ${ }^{3}$ Deidre Hunter, ${ }^{1}$ \\ Earl J. O’Neil, Jr., ${ }^{4}$ Edward J. Shaya, ${ }^{5}$ William A. Baum, ${ }^{6}$ Bel Campbell, ${ }^{7}$ Arthur Code, ${ }^{8}$ \\ Douglas G. Currie, ${ }^{5}$ S. P. Ewald, ${ }^{9}$ J. JefF Hester, ${ }^{10}$ T. Kelsall, ${ }^{11}$ Tod R. Lauer, ${ }^{4}$ \\ Roger Lynds, ${ }^{4}$ Donald P. Schneider, ${ }^{12}$ P. Kenneth SeidelmanN, ${ }^{13}$ AND \\ JAMES A. WestPhaL ${ }^{14}$ \\ Received 1990 November 2; accepted 1990 November 26
}

\begin{abstract}
We describe the prospects for the use of the Wide-Field/Planetary Camera (WFPC) for stellar photometry. The large halos of the point-spread function (PSF) resulting from spherical aberration and from spatial, temporal, and color variations of the PSF are the main limitations to accurate photometry. Degradations caused by crowding are exacerbated by the halos of the PSF. Here we attempt to quantify these effects and determine the current accuracy of stellar photometry with the WFPC. In realistic cases, the brighter stars in crowded fields have 0.09 mag errors; fainter stars have larger errors depending on the degree of crowding. We find that measuring Cepheids in Virgo Cluster galaxies is not currently possible without inordinate increases in exposure times.

Subject headings: image processing — photometry
\end{abstract}

\section{INTRODUCTION}

Stellar photometry projects comprise a significant fraction of the planned $H S T$ scientific programs with the Wide-Field/ Planetary Camera (WFPC). The accuracy of stellar photometry is significantly affected by spherical aberration. In this paper we present a preliminary assessment of the quality of photometric measurements that can be expected from WFPC observations. Although significant improvements over current techniques may be possible, especially with more calibration data and better algorithms, current interest in restructuring WFPC programs warrants a progress report.

\footnotetext{
1 Lowell Observatory, 1400 West Mars Hill Road, Flagstaff, AZ 86001.

2 Physics Department, Jadwin Hall, Princeton University, Princeton, NJ 08544.

${ }^{3}$ UCO/Lick Observatories, Board of Studies in Astronomy and Astrophysics, University of California, Santa Cruz, CA 95064.

${ }^{4}$ National Optical Astronomy Observatories, Box 26732, Tucson, AZ 85726. The National Optical Astronomy Observatories are operated by the Association of Universities for Research in Astronomy, Inc., under cooperative agreement with the National Science Foundation.

5 Department of Physics and Astronomy, University of Maryland, College Park, MD 20742.

${ }^{6}$ Astronomy Department, FM-20, University of Washington, Seattle, WA 98105.

7 Physics Department, University of New Mexico, 800 Yale Boulevard, NE, Albuquerque, NM 87131.

${ }^{\overline{8}}$ Washburn Observatory, University of Wisconsin, 475 North Charter Street, Madison, WI 53706.

${ }^{9}$ Space Telescope Science Institute, 3700 San Martin Drive, Baltimore, MD 21218.

${ }^{10}$ Infrared Processing and Analysis Center, MS 100-22, California Institute of Technology, Pasadena, CA 91125.

11 Code 685.0, Goddard Space Flight Center, Greenbelt, MD 20771.

12 Institute for Advanced Study, Olden Lane, Building E, Princeton, NJ 08540 .

${ }^{13}$ US Naval Observatory, National Almanac Office, Washington, DC 20390.

14 Division of Geological and Planetary Sciences, MS 170-25, California Institute of Technology, Pasadena, CA 91125.
}

\section{OBSERVATIONS}

\subsection{Objects}

We consider observations of five objects obtained as part of the Science Assessment Program: NGC 188, NGC 1850, NGC 925, NGC 6725, and 30 Doradus.

The sparse open cluster NGC 188 was observed twice (on 1990 August 16 and September 19) with the WFC and F555W and F785LP filters with exposure times of $12 \mathrm{~s}$; the current analysis uses only the F555W exposures. Because the spacecraft roll changed between the exposures, stars near the center of the field moved very little but stars at the edge moved by up to 400 pixels $\left(40^{\prime \prime}\right)$.

The young LMC cluster NGC 1850 was likewise observed twice, on 1990 August 16 and September 20. WFC exposures of 10,100 , and $2 \times 1100 \mathrm{~s}$ were taken in both F555W and F785LP. HST pointing and roll were deliberately altered between observations to assess the effects of star placement on the CCDs. In NGC 1850, our analyses have concentrated on a small region, chosen in such a way that it was observed on both dates and contains moderately crowded areas as well as reasonably isolated stars. One of the CCD frames of NGC 1850 is shown in Figure 1 (Plate L8), with the small region marked.

NGC 925 is a nearby spiral galaxy with redshift $792 \mathrm{~km} \mathrm{~s}^{-1}$ which was chosen to assess the feasibility of Cepheid photometry. Two F555W exposures of $1800 \mathrm{~s}$ each, shifted by about $30^{\prime \prime}$, were obtained with the WFC on August 16; each of these was split in half to facilitate removal of cosmic rays. Both exposures were located about $2^{\prime}$ northwest of the center of the galaxy.

NGC 6752 is a globular cluster which contains one of two fields to be used for the photometric calibration of the WFPC with the ground-based photometry of Harris et al. $(1988,1990)$. Only one set of rather underexposed images in filters F555W and F785LP has been obtained to date.

The 30 Doradus images were taken with the PC in F368M 


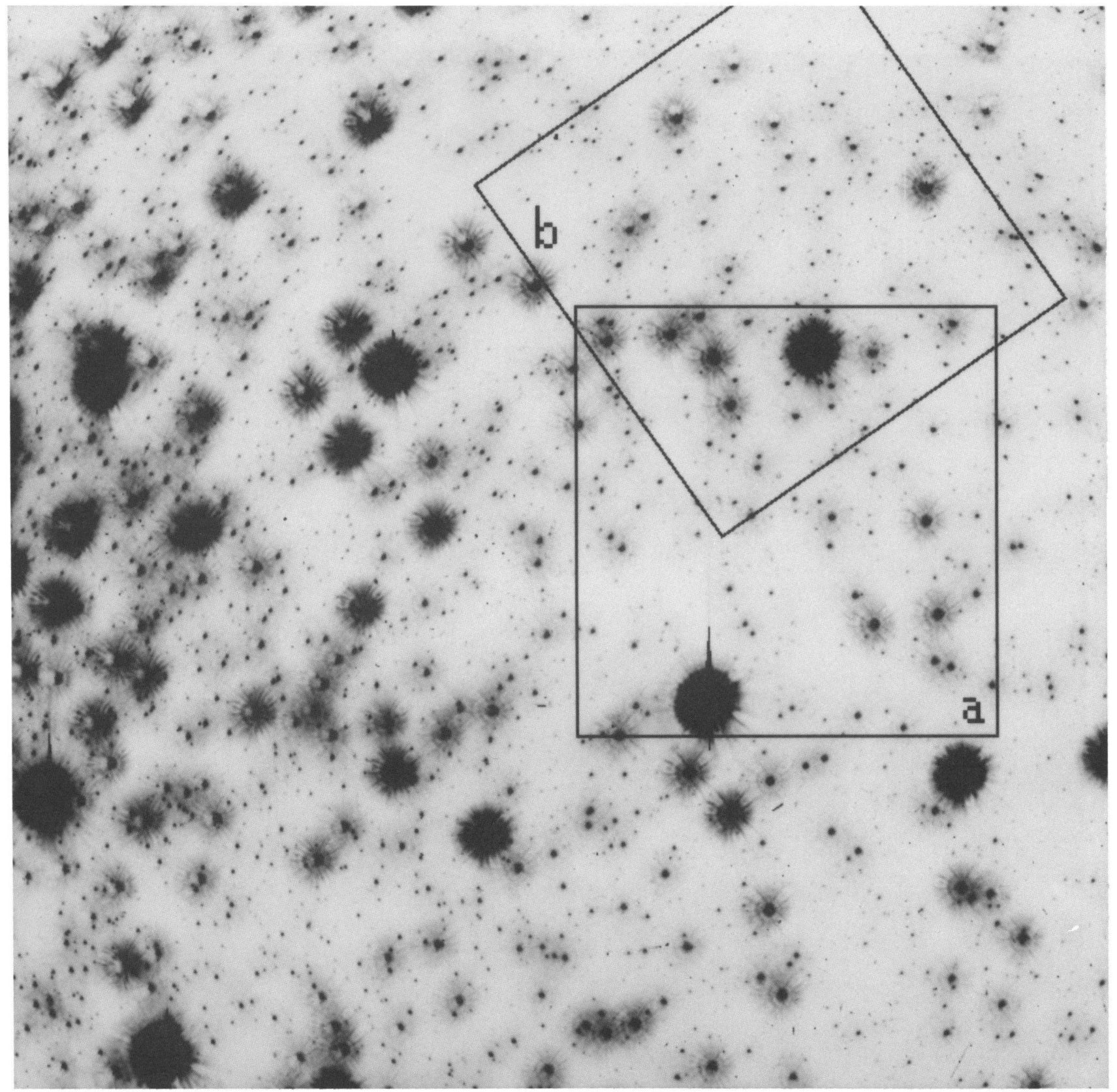

FIG. 1.-1100 s F555W frame of NGC 1850 , taken with the WFC (chip 1). The entire CCD frame is shown, about $75^{\prime \prime} \times 75^{\prime \prime}$. The region selected for photometry is shown in box $a$. Box $b$ indicates the location (relative to the chip) of the same set of stars at the second pointing.

HoltZMan et al. (see 369, L35) 
and F547M. Five consecutive exposures were made in each filter, allowing assessment of short-term point-spread function (PSF) variations. In addition, we use these data to test photometry on deconvolved images.

\subsection{Reduction}

The WFPC and its expected performance are described by Griffiths (1990); it is assumed that the reader is familiar with the instrument. Lauer (1989) has detailed the overall reduction plan required to remove instrument artifacts from WFPC images, including (1) amelioration of the analog-to-digital conversion (ADC) problem, (2) bias and preflash subtraction, (3) dark-current and hot pixel removal, (4) correction for residual image, (5) normalization with a flat field, and (6) removal or tagging of cosmic-ray events. There is at present no evidence to suggest that the overall reduction sequence needs to be changed. Ground-determined ADC corrections and preflash frames have been used, and bias values have been determined from the overscan regions of the on-orbit observations. Dark current is negligible. No correction has been applied for residual image.

The largest problem to date has been flat-fielding. The CCDs suffer from "quantum efficiency hysteresis": the effective quantum efficiency of a pixel depends on its previous exposure history. Variations amounting to $\pm 10 \%$ have been observed in the corners of the on-orbit flats for some filters; these appear to be associated with previous overexposure of the CCDs. These effects can be removed by flooding the CCDs with UV light, but this has not yet been performed on orbit.

Additional uncertainties arise because the Earth "streak flats" used for filters F555W and F785LP were taken through a neutral density filter whose field variation is not yet well known. The filter also has small pinholes, causing dim pupil images to appear in the flat fields. By various intercomparisons, we estimate the $a$ priori flat-field inaccuracies to be about $4 \%$ rms at a typical position; photometry of stars in different regions, to be discussed below, confirms this estimate. Improved flats should be available after the cameras are UVflooded.

Another problem is the existence of large numbers of charged particle detections, hereafter called cosmic-ray events. Cosmic rays usually affect only a few pixels, although they occasionally leave a streak across many pixels. The rate of events is about $2.2 \mathrm{~s}^{-1}$ per CCD in the WFC, so large numbers are seen on long exposures. For all the images presented here, cosmic-ray removal was done by hand, and affected pixels were replaced with the median of surrounding pixels. The longest exposures are equally split in order to facilitate identification and removal of cosmic rays.

\section{THE POINT-SPREAD FUNCTION AND ITS IMPLICATIONS}

Several PSF features relevant to stellar photometry are summarized here and discussed in more detail in subsequent sections.

First, the PSF resulting from spherical aberration is very extended, with light reaching out to a radius of about $3^{\prime \prime}$ (see Burrows et al. 1991). This produces a loss in limiting magnitude and signal-to-noise ratio, increased difficulty in determining total counts, and increased difficulty of detecting and measuring the brightnesses of stars in crowded fields. Additionally, the structure in the halos makes detection of faint sources problematic, because of increased noise from photon statistics and confusion of true sources with PSF structure.
Second, the PSF varies significantly across the field in both the WFC and the PC. Part of this variation arises from some design astigmatism, especially in the WFC, but the major portion arises from vignetting by the secondary mirrors in the repeater optics of the WFPC. The vignetted portion of the pupil varies with field angle; with perfect $H S T$ optics this would not be significant, but with spherical aberration, variable vignetting of the out-of-focus light leads to large PSF variations. Examples can be seen in Figures 2 and 3 (Plate L9), which show closeups of several stars in various regions of the field for both the WFC and the PC. The off-center vignetting by the secondary is visible as the "hole" in the corner images. The detailed structure of the "tendrils" and rings changes as pupil vignetting varies. Figure 1 also shows how the PSF varies regularly over the field in WF1. By design, the obscuration pattern should be centered on the middle of the chip, but in fact there is a systematic offset to the lower right, which may be caused by a tilt of the WFPC pyramid. The offset varies slightly from chip to chip. Clearly, these variations create problems for any stellar photometry scheme which assumes a constant PSF.

Within a given filter, the PSF varies with stellar color, especially for the broad-band filters used in the observations discussed here. However, our photometry has not reached the level of accuracy required to detect color terms related to PSF variations.

Finally, there is preliminary evidence that the PSF varies as a function of time. Analysis of a bright star in the series of 30 Doradus exposures shows that the image structure is clearly varying over the course of one orbit. Aperture growth curves in NGC 188 also suggest a PSF change, in this case over a 5 week period. The level of this variation is not large and resembles a focus change of the telescope. The cause and temporal behavior of this effect are unknown.

Following King (1983), we can calculate the statistical limits on the accuracy of stellar photometry assuming that the PSF, throughput, detector noise, and background level are perfectly known. With these assumptions, the expected $1 \sigma$ magnitude error as a function of apparent magnitude has been calculated using accurate models of the existing and design HST PSF. Figure 4 shows results for an 1100 s exposure in F555W for both the WFC and the PC. The curves are calculated with the predicted unflooded throughput for $H S T$ and WFPC, which has been determined to be correct to within $20 \%$ (cf. $\S 7$ ); a gain of 7.5 electrons $\mathrm{DN}^{-1}$ was used. The curves assume optimal pixel weighting and perfectly known background and thus represent the highest attainable accuracy.

The calculations predict a severe loss in signal-to-noise ratio with the current PSF, amounting to a decrease in limiting magnitude of about 2 mag. We show below that the loss in crowded fields is even greater, owing to uncertainties in determining the background level under faint stars.

Photometric zero-point determination is also severely affected by the aberration. Since the PSF extends to about $3^{\prime \prime}$, very large apertures must be used to measure the total light. However, this requires background values of exquisite accuracy. For example, on a well-exposed star in the WFC with peak value of $3000 \mathrm{DN}$ (total $\approx 60,000 \mathrm{DN}$ ), $1 \%$ accuracy requires that the background be determined to $\pm 0.2 \mathrm{DN}$. The required accuracy for fainter stars is proportionally higher.

In practice, it will not be possible to measure a precise zero point for each CCD frame because most frames will not contain a sufficiently well isolated bright star. Consequently, 
PLATE L9

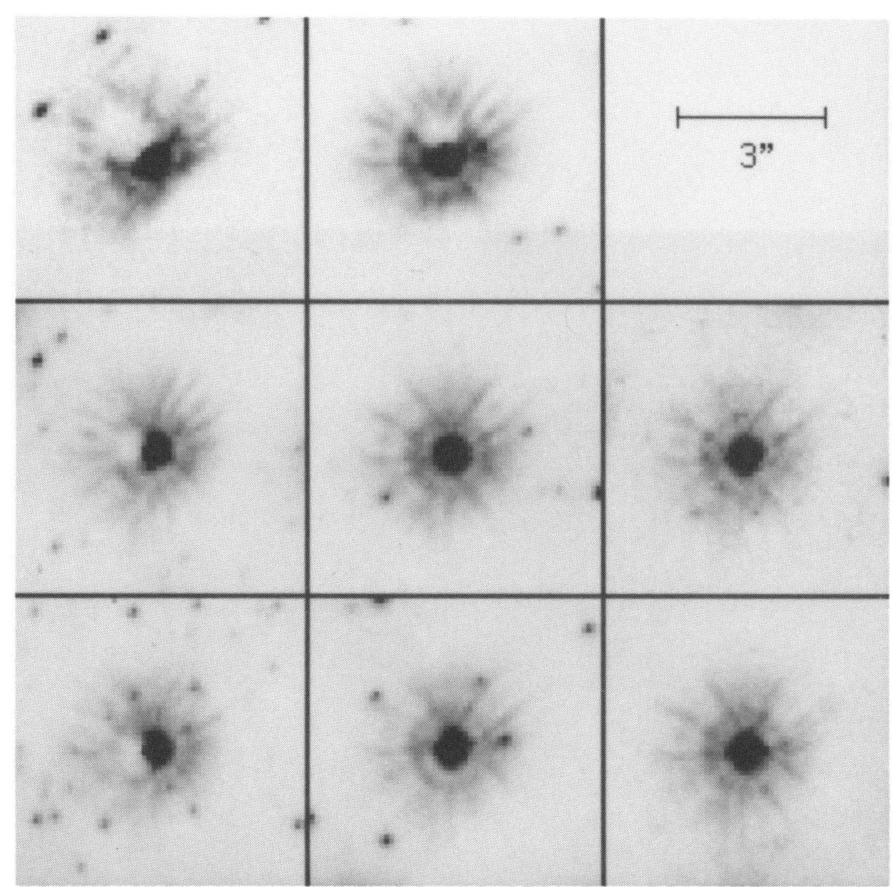

FIG. 2.-Montage of observed PSFs from various field locations in chip WF1 of the WFC and filter F555W. The location of a star in the figure schematically represents its location in the CCD; e.g., the central star is indicative of the PSF in the center of the chip. The Optical Telescope Assembly (OTA) axis is toward the lower left. There was no suitable star in the top right-hand part of the chip; the length of the bar in this panel is $3^{\prime \prime}$.

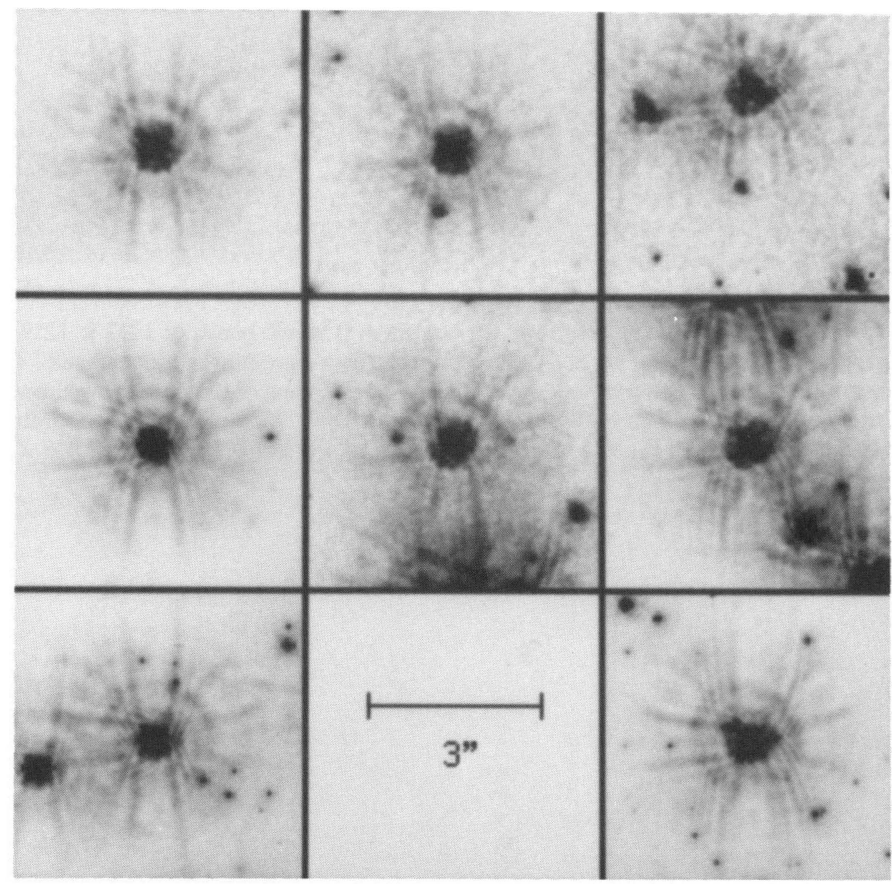

FIG. 3.-Montage of observed PSFs from various field locations in chip P6 of the PC and filter F547M. The location of a star in the figure schematically represents its location in the CCD; e.g., the central star is indicative of the PSF in the center of the chip. The OTA axis is toward the lower left. There was no suitable star in the lower middle part of the chip; the length of the bar in this panel is 3 ".

Holtzman et al. (see 369, L36) 


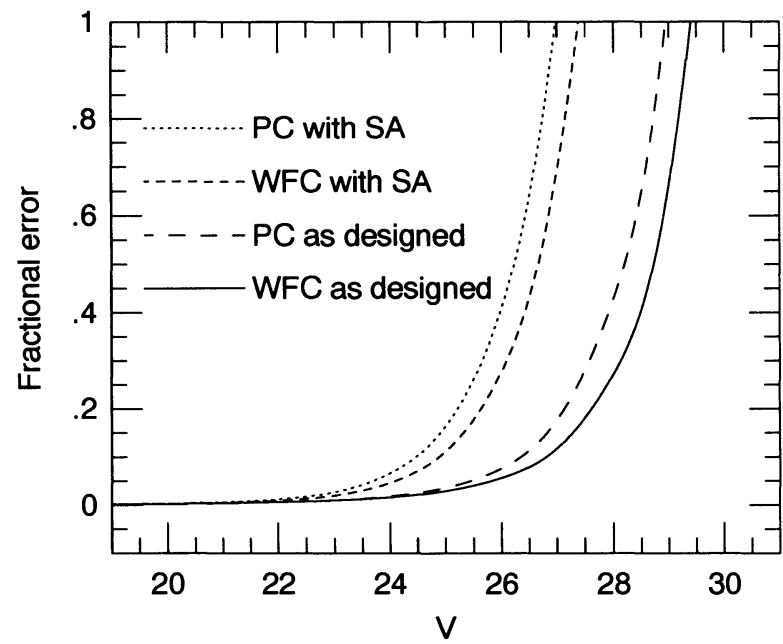

FIG. 4.-Ideal-case best errors for PSF fitting photometry. These curves were computed assuming the expected WFPC/HST throughput and background level, for an $1100 \mathrm{~s}$ exposure in F555W. The four curves represent expected errors for both the WFC and the PC as designed and as currently operating with $H S T$ spherical aberration (SA). A perfectly known background level has been assumed.

the accuracy of a zero point will depend on the constancy of brightness in a small aperture relative to total brightness. This will have some uncertainty due to spatial variations of the PSF as well as temporal variations.

The curves in Figure 4 show the ideal case, where the only limits to photometric accuracy are photon and read-noise statistics. In realistic situations, other effects dominate. These are summarized in Table 1 together with our estimate of the size of the effect. Further details and description are given in subsequent sections.

\section{APERTURE PHOTOMETRY}

The NGC 188 frames were analyzed to determine the current photometric accuracy for isolated stars. Errors were estimated by comparing results from the two exposures and by comparison with ground-based CCD photometry.

\subsection{Background Errors}

We experimented with three different backgroundmeasuring routines: modal routines in DAOPHOT (Stetson 1987) and VISTA (Lauer, Stover, \& Terndrup 1983) and a simple mean. Background accuracy was assessed by running aperture photometry on test patches devoid of obvious objects and cleaned of cosmic rays. Both modal techniques failed badly, setting the background level too low, but the straight mean gave good results with zero bias. Mean background

TABLE 1

Current Stellar Photometry Errors for the WFC

\begin{tabular}{ccc}
\hline \hline \multicolumn{1}{c}{ Error Source } & $\begin{array}{c}\text { Error } \\
(\mathrm{rms} \text { mag) }\end{array}$ & $\begin{array}{c}\text { Text } \\
\text { Section }\end{array}$ \\
\hline Flat field $\ldots \ldots \ldots \ldots \ldots \ldots \ldots \ldots \ldots \ldots \ldots \ldots \ldots \ldots \ldots$ & 0.04 & 4.3 \\
Spatial PSF variations: & & \\
Core-halo ratio $\ldots \ldots \ldots \ldots \ldots \ldots \ldots \ldots \ldots \ldots \ldots$ & 0.05 & 4.2 \\
Shape errors, background fixed $\ldots \ldots \ldots \ldots \ldots \ldots$ & 0.03 & 5.1 \\
Shape errors, background fitted $\ldots \ldots \ldots \ldots \ldots \ldots$ & 0.08 & 5.2 \\
Total shape errors $\ldots \ldots \ldots \ldots \ldots \ldots \ldots \ldots \ldots \ldots \ldots$ & 0.08 & 5.3 \\
Crowded-field background errors $\ldots \ldots \ldots \ldots \ldots \ldots$ & Variable & 5.4 \\
\hline
\end{tabular}

values were adopted. Measuring a mean value to high accuracy is not feasible in any but the sparsest fields.

The rms errors of the test patches were consistent with an equivalent random noise of 17-20 electrons per pixel (aperture radii between 15 and 50 pixels). This level is plausibly accounted for by readout noise (13 electrons rms), preflash ( 8 electrons rms), bias irregularities, unsubtracted cosmic rays, faint stars and galaxies, and large-scale, low-level CCD variations. The diffuse background level is approximately $0.2 \mathrm{DN}$ or less in these exposures.

\subsection{Core-Halo Ratio Errors}

Spatial variations in the PSF perturb aperture photometry, even for aperture radii up to 30 pixels in the WFC; differences as large as $\pm 0.1 \mathrm{mag}$ are seen at small radii, declining to less than 0.01 mag beyond 30 pixels. Effects in the PC should be smaller. Surprisingly, growth curves are more compact when the PSF is poorer (i.e., more asymmetric). Variable vignetting, discussed in $\S 3$, reduces halo light but leaves the core largely unaffected (see Figs. 1-3); hence the PSF becomes relatively more compact. In additon, at extreme field locations there is further halo vignetting at the outermost zones of the pupil from the hole in the repeater primary. Dividing by the flat field removes throughput variations due to vignetting, but this correction is valid only for the total starlight, not for the core and halo separately. This means that there are errors for the core light alone, amounting to the scatter seen at small radii (4 pixels) in the aperture growth curves, or approximately 0.05 mag rms in the WFC (smaller in the PC). We refer to this effect in Table 1 as the core-halo ratio. Such errors affect both smallaperture photometry and small-radius PSF fitting (e.g., DAOPHOT). Since the error is produced by variable vignetting, it should be temporally stable (if the PSF is temporarlly stable) and measurable if sufficient calibration data are obtained. However, a zero-point offset can be produced by temporal variations in the core-halo ratio; for example, the change between the two NGC 188 exposures was $0.12 \mathrm{mag}$ within a 4 pixel radius and 0.08 mag within 15 pixels.

\subsection{Flat-Field Errors}

Flat-field errors have been measured for the WFC in F555W by comparing photometry on shifted frames in which stars have moved by a significant fraction of the width of the chip. The optimum aperture is 15 pixels; at smaller radii, core-halo ratio errors become important; at larger radii, errors in the background level dominate. Flat-field errors are currently about $0.04 \mathrm{mag}$ rms, consistent with $a$ priori errors estimated in $\S 2$.

\subsection{Comparison with Ground-based Photometry}

Caputo et al. (1990) have published $B$ - and $V$-band CCD photometry for a section of NGC 188. About three-quarters of our field overlaps a region observed by them. There are 34 stars in common, with $13.8 \mathrm{mag}<V<17 \mathrm{mag}$. A comparison of our aperture photometry (15 pixel radius) with the groundbased $V$ photometry is presented in Figure $5 a$. The rms difference is $0.05 \mathrm{mag}$, consistent with the observed flat-field errors. No color term is detected.

\section{CROWDED FIELDS: POINT-SPREAD FUNCTION FITTING PHOTOMETRY}

For PSF fitting photometry, we have used the DAOPHOT package (Stetson 1987) with minor modifications to improve 


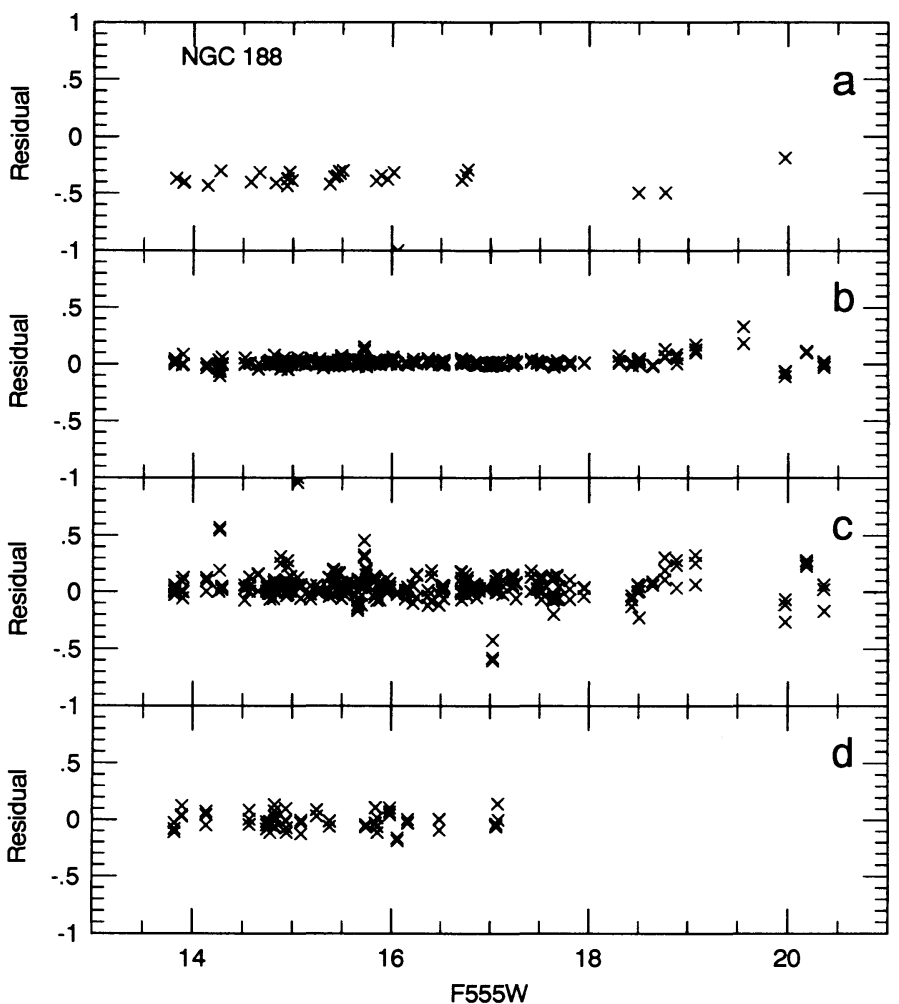

Fig. 5.-F555W photometry of NGC 188. (a) Difference between aperture photometry (15 pixel radius) and ground-based $V$-band CCD photometry (Caputo et al. 1990). (b) Difference between small-aperture photometry (4 pixel radius) and PSF fitting photometry, using a mean background level from an annulus around each star. (c) The same difference, except that the PSF fitting also solves for the background level. (d) Difference between large-aperture photometry (25 pixel radius) and PSF fitting photometry with the background fit. The magnitude coordinate in this and succeeding figures is the F555W magnitude system of Harris et al. $(1988,1990)$. Within a small color term, this system is similar to Johnson $V$.

the results for undersampled data (Holtzman 1990). We have generally used a 3 pixel radius for the PSF fit, and have experimented with two methods of background determination: fitting the background simultaneously with the PSF and determining a modal value in an annulus around each star.

\subsection{PSF Shape Errors: Background Fixed}

Several features complicate PSF fitting, even of isolated stars. The variable shape of the PSF can introduce systematic and random errors dependent on field location if a single PSF is used to reduce the frame. These errors result directly from the mismatch in shape, which leads to an incorrect normalization, and indirectly from an error in the background level if the background is fitted simultaneously. In addition, undersampling of the core of the PSF, especially in the WFC, can introduce systematic errors for stars located at different fractional pixel positions (Holtzman 1990).

We assess direct shape errors by comparison with aperture photometry of isolated stars on a known background. Figure $5 b$ shows such a comparison for NGC 188 . Fits were performed with three different PSFs (from three stars in the image), and all three measurements for each star are shown in the figure. The accurate background level from aperture photometry was used in the PSF fitting photometry. The aperture photometry used a radius of 4 pixels, so that both methods are sensitive to the same light. The scatter is small-only $0.03 \mathrm{mag}$ rms as listed in Table 1.

\subsection{PSF Shape Errors: Background Fit}

Errors increase when the background is not known a priori; in crowded fields, our only viable technique at present is to fit for the background as a free parameter with each star or stellar group. This approach induces magnitude errors even for isolated stars, as shown in Figure $5 c$. Here we repeat the comparison between fitting and small-aperture photometry on NGC 188 , but, instead of using aperture background levels, we fit for the background and stellar brightness simultaneously. The errors increase to $0.08 \mathrm{mag} \mathrm{rms}$ (listed in Table 1), independent of brightness.

\subsection{Total Shape Errors}

Figure $5 d$ shows a comparison between the PSF-fit magnitudes (background fit) and large-aperture magnitudes (25 pixel radius). Here, the rms is $0.08 \mathrm{mag}$, which is listed as the total shape error in Table 1. This error is the "bottom line" for PSF fitting of bright stars, since it is a measure of how well the PSF-fit magnitude agrees with the total light. The total shape error is induced by regular variations of the PSF over the field, so the error listed in Table 1, like the flat-field error, applies only when the full field of the WFC is used. If smaller regions are analyzed, the scatter should be reduced.

\subsection{Crowded-Field Background Errors}

We turn now to the more realistic case of PSF fitting in crowded fields. This case includes all sources of error previously described and, in addition, a serious background problem caused by spherical aberration. The overlapping halos of bright stars produce a sea of background light which contains within it small-scale structure due to the tendrils and rings of the PSF. Moreover, this structure cannot easily be modeled, since the exact morphology of the tendrils, rings, and vignetting is variable over the field (cf. Figs. 2 and 3 ).

Such a situation is not easily quantified; perhaps the best way of understanding the problems is to examine real data. Figure 6 shows the results of two tests on the small region in NGC 1850 marked in Figure 1. Figure $6 a$ shows the differences

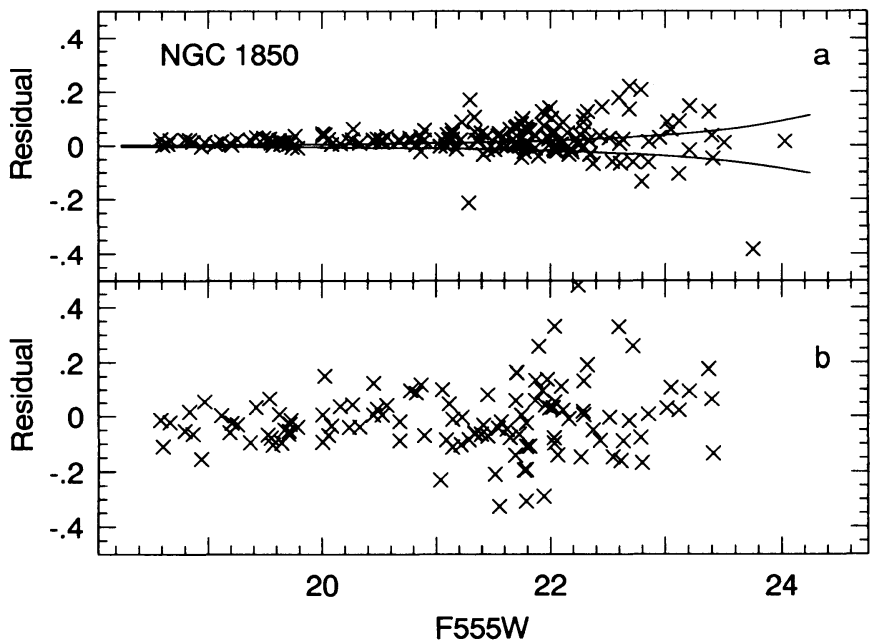

FIG. 6.-F555W photometry of NGC 1850 in a region marked in Fig. 1. (a) Comparison of PSF fitting photometry from two successive $1100 \mathrm{~s}$ exposures, where the registration is shifted by only a small fraction of a pixel. The solid lines show the expected $1 \sigma$ error if the profile and sky level were known exactly. (b) Comparison of the results from two $1100 \mathrm{~s}$ observations of the same field with different pointings so the stars fall in a different location on the CCD array. Stars have moved by about $20^{\prime \prime}$, and the changes in the PSF are moderate. 
in PSF-fit magnitudes (background fit simultaneously) for a pair of $1100 \mathrm{~s}$ cosmic-ray split exposures. These exposures are registered to within a fifteenth of a pixel and were reduced identically, so the test is sensitive to the effects of residual cosmic rays and photon noise. The lines in the figure are the expected $1 \sigma$ errors for the ideal case as in Figure 4, but using the actual mean background level in the image. There is a small, 0.02 mag offset between the two data sets. This effect is not seen in the other cosmic-ray pair and is not understood. Moreover, the scatter exceeds the ideal case, especially at fainter magnitudes. Since flat-field errors and PSF shape variations are the same for both exposures, we attribute the increased scatter to an effect of the crowded-field background.

The second test, in Figure $6 b$, compares the differences in PSF-fit magnitudes for two shifted exposures (regions marked in Fig. 1). In principle, this test incorporates all sources of error: crowding, flat-field errors, shape errors, photon noise, and residual cosmic rays. The scatter for the brighter stars, for which crowding errors are negligible, is only $0.06 \mathrm{mag}$. We attribute this small scatter to the fact that only a small region of the frame has been used. Had we analyzed the whole frame, we would have expected $\approx 0.13$ - the quadrature sum of the total shape and flat-field errors times $\sqrt{ } 2$. The scatter increases to 0.15 mag for the fainter stars where crowded-field background errors become important.

A similar test is shown in Figure 7. Here we use the two shifted frames of NGC 925, each frame being the sum of two $900 \mathrm{~s}$ exposures. The rms scatter in the magnitude differences is $0.19 \mathrm{mag}$ for the brighter stars (F555W $\lesssim 23.1 \mathrm{mag}$ ), increasing to $0.31 \mathrm{mag}$ at fainter magnitudes. This scatter is qualitatively similar to, but larger than, that for the NGC 1850 data, perhaps because large areas of the chips are used and the shift between frames is 300 pixels.

\subsection{Fit versus Modal Background Estimates}

Figure 8 presents two color-magnitude diagrams for NGC 1850 constructed with PSF fitting photometry; in Figure $8 a$ the background is determined as part of the fit as before, while in Figure $8 b$ a modal background estimator is used in an annulus (radius 4-7 pixels) around each star. The fitting tech-

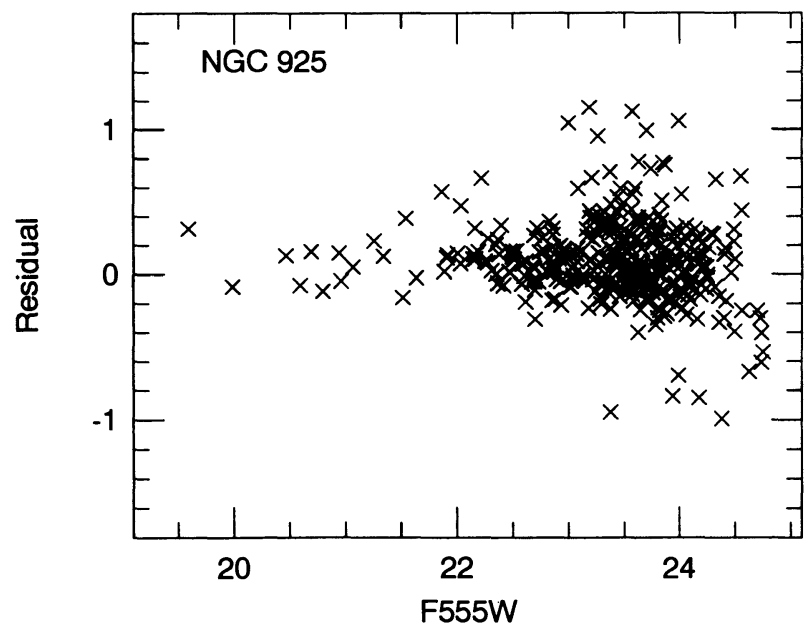

Fig. 7.-F555W photometry for NGC 925. The differences between PSF-fit magnitudes for the two $1800 \mathrm{~s}$ exposures are shown. The data are from WF 3 and WF4, and there is a 300 pixel shift between the exposures. The entire overlap regions in each chip are included.

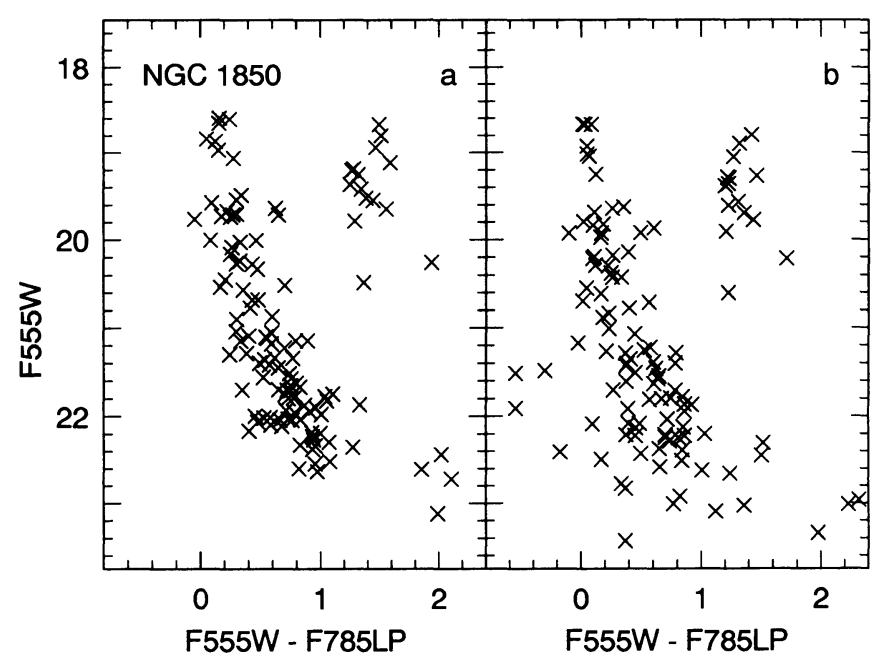

Fig. 8.-Observed color-magnitude (F555W - F785LP vs. F555W) diagram for the region of NGC 1850 marked in Fig. 1, based on one $1100 \mathrm{~s}$ frame each in F555W and F785LP. The magnitude zero points are discussed in the text. (a) Results when the background level is determined during the fitting process. (b) Results for the same field when the background is determined from the modal value in an annulus (4-7 pixels) around each star.

nique is clearly better than the modal estimator. We experimented with changing the annulus size and with aperture magnitudes but could find no technique that produced a colormagnitude diagram as good as that in Figure $8 a$.

\subsection{Star Detection and Limiting Magnitude}

Along with photometric errors, the magnitude limit and the completeness with which stars can be found are of interest. The NGC 1850 and NGC 925 observations provide realistic test cases. The faintest stars in NGC 925 were found by inspection after one round of star subtraction using DAOPHOT, and have a peak count of about $20 \mathrm{DN}$. All areas of the frame were surveyed. Star detection in NGC 1850 was also done manually, but in this case, only clear areas, well away from the visible halos of bright stars, were searched for faint stars. Only a small fraction of the area was thus accessible, and the faintest magnitudes are quite incomplete.

In NGC 925 the faintest measured stars have F555W magnitudes $\approx 24.5 \mathrm{mag}$, whereas the faintest in NGC 1850 are around 22.7 mag. NGC 1850 could have been searched more deeply, and it is believed that stars could have been measured down to $\approx 23.7 \mathrm{mag}$. The remaining difference in limiting magnitude between NGC 1850 and NGC 925 can plausibly be attributed to the longer exposure time and lower degree of contamination by bright stars in NGC 925.

\section{OTHER APPROACHES TO STELLAR PHOTOMETRY}

In an effort to reduce the effects of extended halos, we tried photometry on deconvolved stellar images. In particular, we used PSF fitting to photometer stars in a crowded-field simulation that had been deconvolved with 40 iterations of the Lucy (1974) algorithm. We also measured an F368M frame of 30 Doradus that had been deconvolved with 160 iterations. In the case of the simulation, the scatter in measured magnitudes was comparable in the deconvolved and original images, although there was a systematic nonlinearity of several tenths of a magnitude over a $4 \mathrm{mag}$ range. For 30 Doradus, however, the errors in the photometry of the deconvolved image, as judged by calculated sigmas and residuals, were worse than those of 
the original image. Peak-to-peak magnitude differences from the two images were $\approx 0.5 \mathrm{mag}$. Moreover, the deconvolved image did not reveal stars that were not already apparent in the original image, and it was significantly noisier. More tests are needed, but our initial results are not encouraging.

Future improvements more likely will result from changes to the PSF fitting algorithms and the accumulation of a better calibration data base. Flat-field errors should be greatly reduced after UV flooding. Total shape errors are not random but vary systematically across the field; in principle, they could be mapped with a sufficiently dense grid of exposures in a sparse field like NGC 188. A more serious stumbling block appears to be the local determination of the background level for faint stars in crowded fields. We have some ideas for changes to the PSF fitting algorithm of DAOPHOT that might yield more accurate background values. Whether this problem can be significantly improved is still an open question.

\section{THROUGHPUT AND ABSOLUTE CALIBRATION}

Analysis of the NGC 188 and NGC 6752 frames shows that the throughput at visible wavelengths, in both the WFC and the PC, is within $20 \%$ of the expected value and is even slightly higher than nominal for the unflooded state of the camera (Griffiths 1985; Brown 1986). Throughput is roughly $50 \%$ of nominal at $9000 \AA$ and $25 \%$ at $2300 \AA$. There are fairly large uncertainties in these estimates, and, in any case, the sensitivities will change after the chips are UV-flooded.

Ground-based calibrated stars in NGC 6752 have been used to determine zero points for F555W and F785LP magnitudes as function of flight DN values. On the ground-based system, we find

$$
\text { F555W (ground) }=-2.5 \log \left(\mathrm{DN} \mathrm{s}^{-1}\right)+23.1
$$

and

$$
\text { F785LP (ground) }=-2.5 \log \left(\mathrm{DN} \mathrm{s}^{-1}\right)+21.6 .
$$

Transformations between these ground systems and standard $U B V R I$ are given by Harris et al. $(1988,1990)$. Since these zero points are based on preliminary analyses and contain many of the errors discussed in previous sections, we conservatively estimate the zero-point errors to be $0.2 \mathrm{mag}$.

\section{SCIENCE ASSESSMENT}

NGC 925 was observed to assess the feasibility of measuring Cepheids in nearby galaxies. Our limiting magnitude in $1800 \mathrm{~s}$ is $V \approx 24.5$. With a distance modulus of $29.9 \mathrm{mag}$ (Tully 1988) we can detect stars down to $M_{V}=-5.4$ in this galaxy. However, based on the observed frequency of Cepheids in M33 (Kinman, Mould, \& Wood 1987), there are almost no Cepheids to our present limit for NGC 925, and we would have to reach $\approx 2$ mag deeper to detect and measure 20 or so Cepheids. The same program on a Virgo Cluster galaxy would require at least a 3 mag increase in limiting magnitude. Clearly, HST in its current condition will not be able to measure Cepheids in the Virgo Cluster.

NGC 1850 is a relatively mild example of the crowded-field stellar photometry programs that were to be attempted with the HST. Figure $8 a$ shows that even with our current accuracy, a well-defined main sequence appears in the color-magnitude diagram. The clump to the right of the main sequence appears to be luminosity class III red giants which are part of the general population of the Large Magellanic Cloud and unrelated to NGC 1850. Our estimate is that we could reach $V \approx 23.7 \mathrm{mag}$ in an $1100 \mathrm{~s}$ exposure for a field as crowded as that analyzed here. This limiting magnitude is well within the capabilities of ground-based telescopes working in uncrowded fields. HST's main advantage relative to the ground is that it can work in more crowded regions. However, completeness and accuracy in very crowded regions are yet to be determined.

Figure 4 shows that the spherical aberration costs 2 mag for isolated, faint stars, assuming a perfectly known point-spread function and background level. Our current results suggest that with realistic conditions the cost is perhaps a magnitude larger and the errors can be considerably greater than that expected from photon and read noise alone. With better algorithms, one might hope for improvements, but the 2 mag from Figure 4 is an absolute limit and cannot be recovered until the spherical aberration is corrected.

This research was supported in part by the Hubble Space Telescope Program through various grants and contracts.

\title{
REFERENCES
}

Brown, R. A. 1986, STScI Newletter, 3,8

Burrows, C. J., Holtzman, J. A., Faber, S. M., Bely, P. Y., Hasan, H., Lynds, C. R., \& Schroeder, D. 1991, ApJ, 369, L21

Caputo, F., Chieffi, A., Castellani, V., Collados, M., Roger, C. M., \& Paez, E. 1990, AJ, 99, 261

Griffiths, R. 1985, WFPC Instrument Handbook, Version 1.0 (Baltimore: STScI)

STS1) 1990, WFPC Instrument Handbook, Version 2.1 (Baltimore: STScI)

Harris, H. C., Baum, W. A., Hunter, D. A., \& Kreidl, T. J. 1990, AJ, submitted

Harris, H. C., Hunter, D. A., Baum, W. A., Jones, J. H., \& Kreidl, T. J. 1988, Report to STScI on Ground Based Observations for the WFPC Photometric Calibration

\author{
Holtzman, J. A. 1990, PASP, 102, 806 \\ King, I. R. 1983, PASP, 95, 163 \\ Kinman, T. C., Mould, J. R., \& Wood, P. R. 1987, AJ, 93, 833 \\ Lauer, T. R. 1989, PASP, 101, 445 \\ Lauer, T. R., Stover, R. J., \& Terndrup, D. M. 1983, The VISTA User's Guide \\ (Lick Obs. Tech. Rept., No. 34) \\ Lucy, L. B. 1974, AJ, 79, 745 \\ Stetson, P. B. 1987, PASP, 99, 191 \\ Tully, R. B. 1988, Nearby Galaxy Catalog (New York: Cambridge Univ. Press)
}

